\title{
Imaging Very Thin Particles in ABF STEM
}

\author{
P.J. Phillips, R.F. Klie \\ Department of Physics, University of Illinois at Chicago, Chicago IL 60607
}

Annular bright field (ABF) scanning transmission electron microscopy (STEM), which allows for the visualization of relatively light atoms ( $\mathrm{Li}, \mathrm{N}, \mathrm{O}$, etc.), was recently introduced [1] as a novel imaging mode to couple with the well known high angle annular dark field (HAADF) STEM mode, which excels at imaging in an approximate $\mathrm{Z}^{2}$ contrast. From directly imaging oxygen to lithium [2] and hydrogen [3] in crystalline specimens, ABF imaging is unquestionably useful for a variety of relevant materials science questions. However, in the limit of a very thin specimen (e.g., a few atomic layers), the ABF and HAADF image pair ceases to be complementary. Although this artifact appears to be irrelevant for the imaging of "thick" specimens, it will be of considerable importance to the communities which analyze, for example, monolayer graphene and very thin catalyst particles, which are discussed below. It is emphasized that an experimentalist performing ABF imaging must simply be aware of the potential for ABF/HAADF discrepancies, although the usefulness of the ABF mode remains unquestioned by the authors.

For the present study, two different catalyst samples were imaged in both HAADF and ABF STEM modes. The pertinent difference between the two samples is the atomic number of the main constituent element, either $42(\mathrm{Mo})$ or $78(\mathrm{Pt})$; alumina was the support material in both cases. All STEM imaging was performed on a probe-side aberration-corrected JEOL JEM ARM-200CF, operated at $200 \mathrm{kV}$ with a convergence semi-angle of $14 \mathrm{mrad}$. Of particular relevance to the present study, it is important to note that the detector configuration of the ARM-200CF allows for simultaneous acquisition of the ABF and HAADF (and low angle ADF, if desired) signals. This enables a direct comparison of ABF/HAADF images without needing to account for the lag time required (e.g., sample drift, specimen damage due to repeated scanning and imaging, etc.) to change the camera length to artificially create an ABF detector, which is sometimes necessary. For computational support, STEM image simulations were performed using the multislice code of Kirkland [4]. Input cells were artificially created which consisted of an "amorphous" carbon support (approximately $6.5 \mathrm{~nm}$ thick) coated with a single layer of hexagonally coordinated Mo or Pt atoms.

A subset of experimental results is presented in Fig. 1. It is immediately obvious that for Mo (upper two panels), the arrowed particle is effectively absent from the ABF image, while clearly visible in the HAADF. In the case of Pt atom clusters (lower panels), the ABF image is merely out of focus, which implies that there is an appreciable dependence on the overall scattering power of the imaged species. To further support this, two edge-on Mo particles (traversing the plan-view particle) are visible in both the $\mathrm{ABF}$ and HAADF images, but as in the Pt case, are slightly out of focus in the former. Detailed results will be discussed as a function of microscope defocus for both the Mo and Pt cases, and computational results will also be presented which largely support the experimental findings. In addition to the relevance of the imaged species, preliminary results have indicated that residual microscope aberrations may play an important role in the discrepancy between ABF and HAADF images.

\section{References}

[1] S.D. Findlay, N. Shibata, H. Sawada, E. Okunishi, and Y. Kondo Appl. Phys. Lett. 95 (2009) 191913.

[2] R. Huang and Y. Ikuhara Current Opinion in Solid State and Materials Science 16 (2012) 31-38.

[3] Y.-J. Kim, R. Tao, R.F. Klie and D.N. Seidman ACS Nano (2013) DOI: 10.1021/nn305029b.

[4] E.J. Kirkland Advanced Computing in Electron Microscopy, 2nd Edition, Springer (2010). 
[5] The UIC JEOL JEM-ARM 200CF is supported by an MRI-R ${ }^{2}$ grant from the National Science Foundation (Grant No. DMR-0959470).

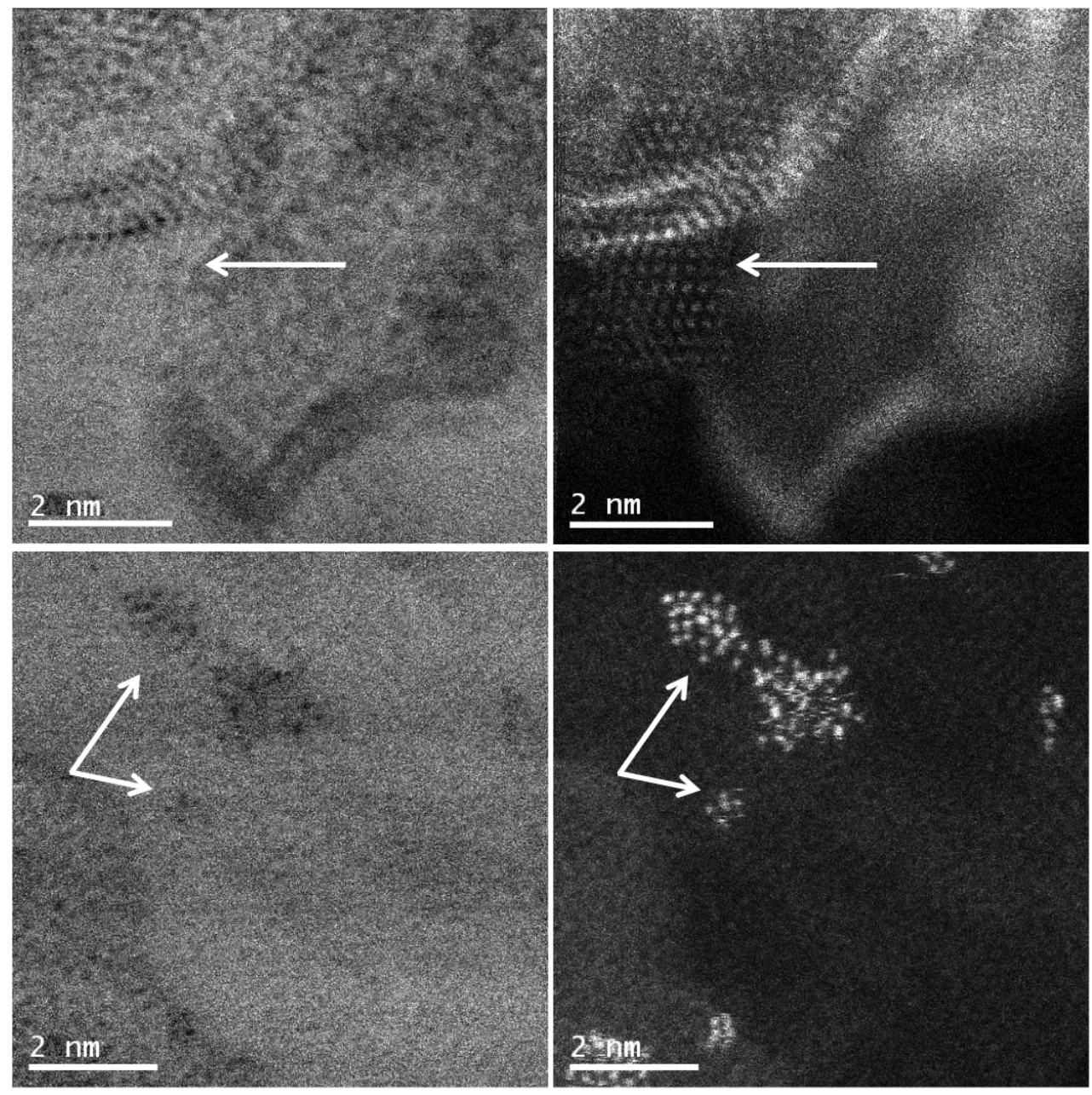

Figure 1: ABF/HAADF STEM image pairs of the Mo (top) and Pt (bottom) catalyst particles. See text for details. 